\title{
Prevalence and Risk Factors of Burden Among Caregivers of Older Patients in the Emergency Department The Caregiver Burden of Acutely Presenting Older Patients
}

\section{Tessel Zaalberg ( $\square$ tzaalberg@viecuri.nl )}

Department of Emergency Medicine, VieCuri Medical Center, Venlo, The Netherlands. https://orcid.org/0000-0002-8947-8304

\section{Dennis Barten}

Department of Emergency Medicine, VieCuri Medical Center, Venlo, The Netherlands.

\section{Caroline van Heugten}

Department of Neuropsychology and Psychopharmacology, Maastricht University, Maastricht, the Netherlands; Limburg Brain Injury Center, Maastricht, the Netherlands; School for Mental Health and Neuroscience, Maastricht University Medical Center, Maastricht, the Netherlands.

\section{Petra Klijnsma}

Department of Geriatric Medicine, VieCuri Medical Center, Venlo, The Netherlands.

\section{Lieve Knarren}

Department of Internal Medicine, VieCuri Medical Center, Venlo, The Netherlands.

\section{Ytje Hiemstra}

Caregiver representative, MantelzorgNL, Zeist, The Netherlands.

\section{Roel Kurvers}

Department of Pediatrics, Maastricht University Medical Center, Maastricht, The Netherlands.

\section{Anita Lekx}

Department of Emergency Medicine, VieCuri Medical Center, Venlo, The Netherlands.

\section{Simon Mooijaart}

Department of Gerontology and Geriatrics, Leiden University Medical Center, Leiden, The Netherlands.

\section{Maryska Janssen-Heijnen}

Department of Epidemiology, VieCuri Medical Center, Venlo, The Netherlands. 9Department of Epidemiology, GROW School for Oncology and Developmental Biology, Faculty of Health Medicine and Life Sciences, Maastricht University Maastricht, The Netherlands

\section{Original research}

Keywords: Emergency department, geriatric emergency medicine, caregiver burden, caregiver strain index 
Posted Date: August 17th, 2020

DOI: https://doi.org/10.21203/rs.3.rs-57953/v1

License: (c) (i) This work is licensed under a Creative Commons Attribution 4.0 International License. Read Full License 


\section{Abstract \\ BACKGROUND}

The number of older individuals that live independently at home is rising. These older individuals often rely on caregivers who typically have a similar age and health status. Therefore, caregivers may experience a high burden. We determined the prevalence and risk factors of burden among caregivers of older patients in the emergency department (ED).

\section{Methods}

a prospective observational cohort study of primary caregivers of patients aged $\geq 70$ years visiting the ED of a Dutch teaching hospital. Structured interviews were conducted with patients and their caregivers. Caregiver burden was measured using the caregiver strain index (CSI). Additionally, data from medical records were extracted to determine potential risk factors. Univariate and multivariate regression analyses were conducted to identify independent determinants for burden.

\section{RESULTS}

A primary caregiver was reported by 200 of 628 included ED patients. The mean age of patients with a caregiver was 82 (SD 6.7) years and the mean age of the caregivers was 66 (SD 12.4) years. Seventyeight caregivers (39\%) experienced a high burden. Multivariate analysis showed a significant association between high caregiver burden and patients with cognitive impairment or dependency for instrumental activities of daily living (IADL) and more self-reported hours of care per day.

\section{CONCLUSION}

Almost $40 \%$ of older patients in the ED have a caregiver who experiences a high burden. Formal assessment in the ED may help provide adequate care to the patients and their caregivers.

\section{Introduction}

Acutely presenting older patients in the emergency department (ED) often have caregivers with similar age and health status, which may result in a fragile balance regarding care needs at home. Surprisingly, information on the prevalence and risk factors of caregiver burden is lacking. Especially little is known about their level of burden when older patients seek acute medical care.

In many developed countries, the number of older individuals who live independently at home is increasing. ${ }^{(1)}$ In 2011, almost half of all older adults in the US reported receiving help with daily activities. Of these, $83 \%$ lived in community settings and nearly 1 in 5 received help with their most basic self-care 
or mobility activities. ${ }^{(2)}$ A recent study in the Netherlands showed that $95 \%$ of older ED patients lived independently at home prior to their visit. A caregiver was reported by $51 \%$ of patients. ${ }^{(3)}$ Overall, $16 \%$ of caregivers of patients with cancer experience a high to severe burden, ${ }^{(4)}$ and for caregivers of stroke survivors and patients with dementia this burden is even higher, approaching $35 \%$ and $60 \%$, respectively. $(5,6)$ Furthermore, the number of hours of care delivered is associated with the occurrence of burnout among caregivers. ${ }^{(7,8)}$ Caregivers with a high burden may cause potential health risks for patients through inadequate care, medication mistakes, and elder abuse or neglect. ${ }^{(9)}$

In this prospective observational cohort study, we determined the prevalence and risk factors of caregiver burden of acutely presenting older patients in the ED.

\section{Methods}

Study design

We conducted a prospective observational cohort study at the ED at VieCuri Medical Center in Venlo, the Netherlands. This is a teaching hospital and a level 2 trauma center with an annual ED census of 25,000 patients. In 2019, 33\% of the patients who presented at the ED were $\geq 70$ years old. In the Netherlands, primary healthcare is well developed and accessible for patients 24 hours a day. General practitioners (GPs) serve as gatekeepers to hospital care. During office hours, patients can consult their own GP, usually obtaining an appointment that day. Afterhours primary care is provided through GPs cooperatives. The majority of ED patients are referred by their GPs or by ambulance. Self-referrals compromise a small minority. ${ }^{(10)}$

This study was approved by the Institutional Review Board of VieCuri Medical Center, Venlo, the Netherlands (\#458).

Participant selection

Participants were recruited between November 6, 2019 and January 19, 2020, with an interruption of one week from December 25 , to January 1 . All patients $\geq 70$ years of age were screened for eligibility by the ED's treating physician. Their baseline characteristics were collected. If patients reported a caregiver, both the patients and the caregiver were included for further analysis. A caregiver was defined according to Kent et al. as "individuals who provide care that is typically uncompensated and usually at home, which involves significant amounts of time and energy for months or years, and which requires the performance of tasks that may be physically, emotionally, socially or financially demanding."(11) Exclusion criteria for patients were living in a nursing home, unwilling to participate, unable to participate due to language barriers, highest triage category (red) using the Manchester triage system, ${ }^{(12)}$ and inability to provide informed consent (with no legal representative available). Patients and their caregivers were provided written information about the study and the treating physician obtained informed consent. 
A structured medical record review of patients was performed in which information was collected to identify potential risk factors for caregiver burden. Patients and caregivers were interviewed separately by one researcher (T.Z.) using questionnaires. The interview took place during the ED visit or within 7 days after the ED visit by telephone.

The following information about patients was collected from the medical record review and the questionnaires: age; sex; marital status; highest level of education (divided into two categories: low [unfinished, primary, secondary] and high education [vocational and tertiary studies]); employment; nationality; reason for ED visit (trauma-related or not); mode of ED referral; cognitive impairment (defined as official diagnosis of dementia determined by a geriatrician); active malignancy; homecare; number of caregivers; age adjusted Charlson Comorbidity Index (ACCI) presented as estimated 10-years survival; (13) Acutely Presenting Older Patients (APOP) score (risk of functional decline or mortality in three months); ${ }^{(14)}$ Activities of Daily Living (ADL) KATZ score ${ }^{(15)}$ and Instrumental activities of daily living (IADL) score.

(16) The ADL-KATZ score ranges from 0 to 6 and a higher score corresponds with higher dependency. The IADL score measures, seven everyday functional competence through self-reporting. Each item could score 2 points. Score ranges from 0-8 (dependent) or 9-14 (independent).

The caregiver information collected included: age; sex; nationality; relationship to the patient; highest level of education (divided into two categories: low [unfinished, primary, secondary] and high education [vocational and tertiary studies]); employment; self- reported number of hours of caring for the patient; distance to the patient; term of care provided; clinical frailty scale (CFS) ${ }^{(17)}$ and caregiver strain index (CSI). ${ }^{(18)}$

The CSI is a brief and easy 13-question tool used to quickly identify potential burden of caregivers that contains subjective and objective elements. Positive responses to $\geq 7$ questions indicate a high level of strain. There is a minimum of one question for each of the following domains: employment, financial, physical, social and time. ${ }^{(18)}$ Caregivers with a CSI index $\geq 7$ completed this questionnaire again four weeks after the initial ED visit to determine whether the burden persisted.

Sample size

We aimed to include 200 caregivers based on previous research with a comparable study setting. $(3,19)$ With an expected burden of $20 \%$, we estimated including 160 caregivers without and 40 caregivers with burden.

\section{Statistical analysis}

SPSS version 24 (International Business Machines Cooperation, Amsterdam, the Netherlands) was used for statistical analyses. Baseline characteristics were presented as numbers and percentages for categorical variables and means or medians for continuous variables. Pearson's chi squared t- test and 
the Mann Whitney u- test were used to determine the association between experienced burden (yes/no) and dichotomous and categorical patient and caregiver characteristics. Univariate logistic regression analyses were conducted to analyze the association between patient and caregiver characteristics as independent variables, and the caregiver burden as dependent variable. A high burden was defined as a $\mathrm{CSI}>7 .{ }^{(20)}$ Characteristics that were associated with burden in univariate analyses were included in a multivariate logistic regression analysis to investigate which characteristics were independently associated with burden. Age and sex of both patient and caregiver were also included in this multivariate regression analysis. We calculated odds ratios (ORs) for burden with $95 \%$ confidence intervals $(\mathrm{Cl})$ and a $p$ value of $<0.05$ was considered significant.

\section{Results}

\section{Patients}

During the study period, 1,086 patients aged $\geq 70$ years visited the ED. Of these, 267 were not screened for inclusion (Fig. 1). Of the remaining 819 patients eligible for inclusion, 628 were included in the analyses. The reasons for exclusion $(n=191)$ were: living in a nursing home $(n=63 ; 33 \%)$, unwilling to participate $(n=20 ; 10.5 \%)$, language barrier $(n=14 ; 7.3 \%)$, highest triage category $(n=47 ; n=24.6 \%)$, inability to provide informed consent $(n=17 ; 8.9 \%)$, or caregiver could not be reached by telephone $(n=$ $30 ; 15.7 \%$ ) (Fig. 1). A primary caregiver was reported by $31.8 \%$ of the 628 study participants $(n=200)$.

Compared to patients without a caregiver, patients with a caregiver were on average older (78 vs 82 years). The baseline characteristics were similar (Table 1). 
Table 1

Baseline characteristics

\begin{tabular}{|llll|}
\hline & & $\begin{array}{l}\text { Patients without a } \\
\text { caregiver } \\
\mathbf{n}=\mathbf{4 2 8}\end{array}$ & $\begin{array}{l}\text { Patients with a } \\
\text { caregiver }\end{array}$ \\
& & $\mathbf{n = 2 0 0}$
\end{tabular}

Patients and their caregivers

Of the patients with a caregiver, $20 \%$ had an active malignancy and $22.5 \%$ were cognitively impaired. The percentage of patients living alone was $42.5 \%$. The mean age (SD) of the caregivers was 66 (12.4) years, $67.5 \%$ were female and $51 \%$ of the caregivers were the spouse or partner of the patient. Among the 200 
caregivers in this study, 78 (39\%) experienced a high burden with a CSI score $\geq 7$. Both the patient age and caregiver age were not significantly associated with burden.

Compared to caregivers with a low burden, caregivers with a high burden were more often caregivers of a patient with cognitive impairment (43.6\% vs $9 \% ; p=0.000)$, a higher ADL score $(20.5 \%$ vs $6.6 \% ; p=0.003)$ and a lower IADL score $(93.6 \%$ vs $63.9 \% ; p=0.000)$. The median number of self-reported hours of care per day by caregivers was significantly higher among caregivers with a high burden, both for partners in the caregiver role (24.00 hours (5.30-24.00) vs 2.00 hours $(1.00-4.00) ; p=0.000)$ and children $(2.00$ hours $(1.00-4.37)$ vs 1.00 hour $(0.34-1.50) ; p=0.000)$ as caregiver.

Patient and caregiver characteristics are summarized in Table 2. 
Table 2

Associations of caregiver burden with patient and caregiver characteristics

\begin{tabular}{|c|c|c|c|c|}
\hline \multicolumn{2}{|l|}{ Patient Characteristics } & $\begin{array}{l}\text { Burden + } \\
n=78\end{array}$ & $\begin{array}{l}\text { Burden - } \\
n=122\end{array}$ & $\begin{array}{l}\mathrm{p}- \\
\text { value }\end{array}$ \\
\hline \multicolumn{2}{|l|}{ Age in years, mean (SD) } & $82.2(6.5)$ & $82.5(6.9)$ & 0.717 \\
\hline \multirow[t]{2}{*}{ Gender (\%) } & Male & $40(51.3)$ & $59(48.4)$ & 0.687 \\
\hline & Female & $38(48.7)$ & $63(51.6)$ & \\
\hline \multicolumn{2}{|l|}{ Living alone (\%) } & $29(37.2)$ & $56(45.9)$ & 0.224 \\
\hline \multicolumn{2}{|l|}{ Active malignancy (\%) } & $13(16.7)$ & $27(22.1)$ & 0.346 \\
\hline \multicolumn{2}{|l|}{$\begin{array}{l}\text { Official diagnosis cognitive } \\
\text { impairment* }(\%)\end{array}$} & $34(43.6)$ & $11(9)$ & 0.000 \\
\hline \multicolumn{2}{|l|}{ Receiving homecare (\%) } & $44(56.4)$ & $56(45.9)$ & 0.147 \\
\hline \multirow[t]{2}{*}{ Estimated 10 years survival (\%) } & $0-50 \%$ & $69(88.5)$ & $98(80.3)$ & 0.131 \\
\hline & $>50 \%$ & $9(11.5)$ & $24(19.7)$ & \\
\hline \multirow[t]{2}{*}{ APOP score (\%) } & $\begin{array}{l}\text { Low risk of adverse } \\
\text { outcome }\end{array}$ & $31(39.7)$ & $64(52.5)$ & 0.079 \\
\hline & $\begin{array}{l}\text { High risk of adverse } \\
\text { outcome }\end{array}$ & $47(60.3)$ & $58(47.5)$ & \\
\hline \multirow[t]{2}{*}{ ADL KATZ (\%) } & $\begin{array}{l}\text { Low level of } \\
\text { dependency }\end{array}$ & \multirow{2}{*}{$\begin{array}{l}62(79.5) \\
16(20.5)\end{array}$} & $\begin{array}{l}114 \\
(93,4)\end{array}$ & 0.003 \\
\hline & $\begin{array}{l}\text { High level of } \\
\text { dependency }\end{array}$ & & $8(6.6)$ & \\
\hline \multirow[t]{2}{*}{ IADL } & Dependent & 73 (93.6) & $78(63.9)$ & 0.000 \\
\hline & Independent & $5(6.4)$ & $44(36.1)$ & \\
\hline \multirow[t]{2}{*}{ Level of education (\%) } & $\begin{array}{l}\text { Low (unfinished - } \\
\text { secondary) }\end{array}$ & $57(73.1)$ & $84(68.9)$ & 0.523 \\
\hline & $\begin{array}{l}\text { High (vocational - } \\
\text { tertiary) }\end{array}$ & $21(26.9)$ & $38(31.1)$ & \\
\hline \multirow[t]{5}{*}{ Arrival } & By ambulance & $22(28.2)$ & $28(23.0)$ & 0.087 \\
\hline & Referred by GP & $22(28.2)$ & $30(24.6)$ & \\
\hline & By ambulance & $24(30.8)$ & $57(46.7)$ & \\
\hline & Own transport & $10(12.8)$ & $7(5.7)$ & \\
\hline & Other & & & \\
\hline \multicolumn{2}{|l|}{ Trauma-related ED visit (\%) } & $24(30.8)$ & $25(20.5)$ & 0.099 \\
\hline
\end{tabular}




\begin{tabular}{|c|c|c|c|c|}
\hline \multicolumn{2}{|l|}{ Patient Characteristics } & $\begin{array}{l}\text { Burden + } \\
n=78\end{array}$ & $\begin{array}{l}\text { Burden - } \\
n=122\end{array}$ & $\begin{array}{l}\mathrm{p}- \\
\text { value }\end{array}$ \\
\hline \multirow[t]{6}{*}{ Specialty (\%) } & Internal medicine & $21(26.9)$ & $29(23.8)$ & \multirow[t]{6}{*}{0.189} \\
\hline & Traumatology & $24(30.8)$ & $25(20.5)$ & \\
\hline & Neurology & 15 (19.2) & $16(13.1)$ & \\
\hline & Surgery & $5(6.4)$ & $16(13.1)$ & \\
\hline & Pulmonary medicine & $11(14.1)$ & $29(23.8)$ & \\
\hline & Other & $2(2.6)$ & $7(5.7)$ & \\
\hline \multirow[t]{5}{*}{ Presenting time (\%) } & \multirow{2}{*}{$\begin{array}{l}\text { Office hours (8.00- } \\
17.00)\end{array}$} & $46(59.0)$ & \multirow{2}{*}{$\begin{array}{l}86 \\
(70.5 \%)\end{array}$} & \multirow[t]{5}{*}{0.335} \\
\hline & & \multirow{2}{*}{$\begin{array}{l}10(12.8) \\
5(6.4 \%)\end{array}$} & & \\
\hline & $\begin{array}{l}\text { Evening hours } \\
(17.01-0.00)\end{array}$ & & $\begin{array}{l}14 \\
(11.5 \%)\end{array}$ & \\
\hline & $\begin{array}{l}\text { Night hours }(0.01- \\
7.59)\end{array}$ & \multirow[t]{2}{*}{$17(21.8 \%)$} & \multirow{2}{*}{$\begin{array}{l}6(4.9 \%) \\
16 \\
(13.1 \%)\end{array}$} & \\
\hline & Weekend hours & & & \\
\hline \multicolumn{5}{|l|}{ Caregiver Characteristics } \\
\hline Age in years, mean (SD) & & $66.1(11.6)$ & $\begin{array}{l}66.4 \\
(13.0)\end{array}$ & 0.846 \\
\hline \multirow[t]{2}{*}{ Gender (\%) } & Female & $56(71.8)$ & $79(64.8)$ & \multirow[t]{2}{*}{0.300} \\
\hline & Male & $22(28.2)$ & $43(35.2)$ & \\
\hline Employment (\%) & & $22(28.2)$ & $48(39.3)$ & 0.107 \\
\hline Caregiver living with patient (\%) & & $45(58.4)$ & $61(50)$ & 0.245 \\
\hline \multirow[t]{2}{*}{ Level of education (\%) } & $\begin{array}{l}\text { Low (Unfinished - } \\
\text { secondary) }\end{array}$ & $30(38.5)$ & $48(39.3)$ & \multirow[t]{2}{*}{0.901} \\
\hline & $\begin{array}{l}\text { High (vocational - } \\
\text { tertiary) }\end{array}$ & $48(61.5)$ & $74(60.7)$ & \\
\hline \multirow[t]{5}{*}{ Relationship with patient (\%) } & Partner & $42(53.8)$ & $61(50)$ & \multirow[t]{5}{*}{0.347} \\
\hline & Children & $25(32.1)$ & $30(24.6)$ & \\
\hline & Daughter & $9(11.5)$ & $23(18.9)$ & \\
\hline & Son & $2(2.6)$ & $8(6.5)$ & \\
\hline & Other & & & \\
\hline
\end{tabular}




\begin{tabular}{|c|c|c|c|c|}
\hline \multicolumn{2}{|l|}{ Patient Characteristics } & $\begin{array}{l}\text { Burden + } \\
\mathrm{n}=78\end{array}$ & $\begin{array}{l}\text { Burden - } \\
n=122\end{array}$ & $\begin{array}{l}\text { p- } \\
\text { value }\end{array}$ \\
\hline \multirow[t]{2}{*}{$\begin{array}{l}\text { Self-reported number of hours of } \\
\text { care/day, median (IQR) }\end{array}$} & Partner & $\begin{array}{l}24.0(5.30- \\
24.00)\end{array}$ & $\begin{array}{l}2.0(1.0- \\
4.0)\end{array}$ & 0.000 \\
\hline & Children & $\begin{array}{l}2.0(1.00- \\
4.37)\end{array}$ & $\begin{array}{l}1.0(0.3- \\
1.5)\end{array}$ & 0.000 \\
\hline \multirow{3}{*}{$\begin{array}{l}\text { Self-reported number of hours or } \\
\text { care/day (\%) }\end{array}$} & $\leq 1$ hour & $12(15.4)$ & $58(47.5)$ & \multirow[t]{3}{*}{0.000} \\
\hline & $1-23$ hours & $37(47.4)$ & $51(41.8)$ & \\
\hline & 24 hours & $29(37.2)$ & $13(10.7)$ & \\
\hline \multirow{2}{*}{$\begin{array}{l}\text { Distance to the patients } \mathrm{km} \text {, median } \\
\text { (IQR) }\end{array}$} & Partner & $0.0(0-0)$ & $0.0(0-0)$ & 0.484 \\
\hline & Children & $1.0(0.28-5)$ & $\begin{array}{l}2.0(1.0- \\
5.5)\end{array}$ & 0.136 \\
\hline \multirow[t]{3}{*}{ Distance to the patients $\mathrm{km}(\%)$} & Living together & $45(57.7)$ & $61(50)$ & \multirow[t]{3}{*}{0.435} \\
\hline & $\leq 10 \mathrm{~km}$ & $29(37.2)$ & $50(41)$ & \\
\hline & $>10 \mathrm{~km}$ & $4(5.1)$ & $11(9)$ & \\
\hline \multirow[t]{2}{*}{$\begin{array}{l}\text { Term of care provided years, median } \\
\text { (IQR) }\end{array}$} & Partner & $\begin{array}{l}5.0(2.4- \\
15.0)\end{array}$ & $\begin{array}{l}5.0(1.5- \\
11.5)\end{array}$ & \multirow{2}{*}{0.260} \\
\hline & Children & $\begin{array}{l}4.0(2.0- \\
10.0)\end{array}$ & $\begin{array}{l}6.0(2.0- \\
10.0)\end{array}$ & \\
\hline \multirow[t]{4}{*}{ CFS (\%) } & Very Fit & $8(10.3)$ & $21(17.2)$ & \multirow[t]{4}{*}{0.493} \\
\hline & Well & $30(38.5)$ & $49(40.2)$ & \\
\hline & Managing Well & $29(37.2)$ & $38(31.1)$ & \\
\hline & Vulnerable & $14(11.5)$ & $11(14.1)$ & \\
\hline \multicolumn{5}{|c|}{ Burden $+=$ Caregiver strain index $\geq 7$. Burden $-=$ Caregiver strain index $<7$} \\
\hline \multicolumn{5}{|c|}{ ED = Emergency Department. SD = Standard deviation; GP = general practitioner; } \\
\hline \multicolumn{5}{|c|}{$\begin{array}{l}\text { APOP }=\text { acutely Presenting Older Patients. An APOP score of } 0-44 \text { is defined as a low risk of adverse } \\
\text { outcomes and an APOP score } \geq 45 \text { is defined as a high risk of adverse outcomes. }\end{array}$} \\
\hline \multicolumn{5}{|c|}{$\begin{array}{l}\text { ADL KATZ score = Activities of Daily living. Dependency of } 0-3 \text { items is defined as a low level of } \\
\text { dependency, and dependency of } 4-6 \text { items is defined as a high level of dependency. }\end{array}$} \\
\hline \multicolumn{5}{|c|}{$\begin{array}{l}\text { IADL score = Instrumental activities of daily living. Dependent is defined as an IADL score } 0-8 . \\
\text { Independent is defined as an IADL score }>8 \text {. }\end{array}$} \\
\hline
\end{tabular}




\begin{tabular}{|lll|}
\hline Patient Characteristics & $\begin{array}{l}\text { Burden }+ \\
\mathbf{n = 7 8}\end{array}$ & $\begin{array}{l}\text { Burden }- \\
\mathbf{n = 1 2 2}\end{array}$ \\
\hline $\begin{array}{l}\text { * Diagnosed by a geriatrician (including dementia, Alzheimer's disease, frontotemporal degeneration, } \\
\text { vewy body disease, traumatic brain injury (TBI) and neurocognitive issues due to Parkinson's } \\
\text { disease). }\end{array}$ \\
\hline
\end{tabular}

In the univariate logistic regression analyses, cognitive impairment $(\mathrm{OR}=7.80), A D L K A T Z$ score with a high level of dependency $(O R=3.68)$, IADL dependency $(O R=8.24)$ and self-reported number of hours of care per day ( $O R=3.51$ for $1-23$ hours vs $<1$ hour and $O R=10.78$ for 24 hours vs $<1$ hour) were significantly associated with a high caregiver burden (Table 3 ). 
Table 3

Univariate and multivariate regression analyses

\begin{tabular}{|c|c|c|c|}
\hline \multicolumn{2}{|l|}{ Patient Characteristics } & $\begin{array}{l}\text { Univariate } \\
\text { regression } \\
\text { OR }(95 \% \mathrm{Cl})\end{array}$ & $\begin{array}{l}\text { Multivariate } \\
\text { regression } \\
\text { OR }(95 \% \mathrm{Cl})\end{array}$ \\
\hline Age (per year) & & $\begin{array}{l}1.01(0.97- \\
1.05)\end{array}$ & $1.01(0.95-1.06)$ \\
\hline \multirow[t]{2}{*}{ Gender } & Female & 1 & 1 \\
\hline & Male & $\begin{array}{l}1.12(0.64- \\
1.99)\end{array}$ & $0.84(0.40-1.78)$ \\
\hline \multirow[t]{2}{*}{ Living alone } & No & 1 & $\mathrm{NI}$ \\
\hline & Yes & $\begin{array}{l}0.70(0.39- \\
1.25)\end{array}$ & \\
\hline \multirow[t]{2}{*}{ Active malignancy } & No & 1 & $\mathrm{NI}$ \\
\hline & Yes & $\begin{array}{l}0.70(0.34- \\
1.47)\end{array}$ & \\
\hline \multirow{2}{*}{$\begin{array}{l}\text { Official diagnosis cognitive } \\
\text { impairment* }\end{array}$} & No & 1 & 1 \\
\hline & Yes & $\begin{array}{l}7.80(3.63- \\
16.75)\end{array}$ & $3.90(1.64-9.30)$ \\
\hline \multirow[t]{2}{*}{ Receiving homecare } & No & 1 & $\mathrm{NI}$ \\
\hline & Yes & $\begin{array}{l}1.53(0.86- \\
2.702)\end{array}$ & \\
\hline \multirow[t]{2}{*}{ Estimated 10 years survival } & $0-50 \%$ & 1 & $\mathrm{NI}$ \\
\hline & $>50 \%$ & $\begin{array}{l}0.53(0.23- \\
1.21)\end{array}$ & \\
\hline \multirow[t]{2}{*}{ APOP score } & $\begin{array}{l}\text { Low risk of adverse } \\
\text { outcome }\end{array}$ & $\begin{array}{l}0.60(0.34- \\
1.06)\end{array}$ & $\mathrm{NI}$ \\
\hline & $\begin{array}{l}\text { High risk of adverse } \\
\text { outcome }\end{array}$ & 1 & \\
\hline \multirow[t]{2}{*}{ ADL KATZ } & $\begin{array}{l}\text { Low level of } \\
\text { dependency }\end{array}$ & 1 & 1 \\
\hline & $\begin{array}{l}\text { High level of } \\
\text { dependency }\end{array}$ & $\begin{array}{l}3.68(1.49- \\
9.08)\end{array}$ & $1.08(0.36-3.29)$ \\
\hline \multirow[t]{2}{*}{ IADL } & Independent & 1 & 1 \\
\hline & Dependent & $\begin{array}{l}8.24(3.10- \\
21.91)\end{array}$ & $4.46(1.50-13.23)$ \\
\hline
\end{tabular}




\begin{tabular}{|c|c|c|c|}
\hline \multirow[t]{2}{*}{ Patient Characteristics } & & Univariate & Multivariate \\
\hline & & OR (95\% Cl) & OR (95\% Cl) \\
\hline \multirow[t]{2}{*}{ Level of education } & $\begin{array}{l}\text { Low (Unfinished - } \\
\text { secondary) }\end{array}$ & 1 & \multirow[t]{2}{*}{$\mathrm{NI}$} \\
\hline & $\begin{array}{l}\text { High (vocational - } \\
\text { tertiary) }\end{array}$ & $\begin{array}{l}0.81(0.43- \\
1.53)\end{array}$ & \\
\hline \multirow[t]{4}{*}{ Presenting time } & $\begin{array}{l}\text { Office hours (8.00- } \\
17.00)\end{array}$ & 1 & \multirow[t]{4}{*}{$\mathrm{NI}$} \\
\hline & $\begin{array}{l}\text { Evening hours (17.01- } \\
0.00 \text { ) }\end{array}$ & $\begin{array}{l}1.34(0.55- \\
3.24)\end{array}$ & \\
\hline & $\begin{array}{l}\text { Night hours }(0.01- \\
7.59)\end{array}$ & $\begin{array}{l}1.59(0.45- \\
5.38)\end{array}$ & \\
\hline & Weekend hours & $\begin{array}{l}1.99(0.92- \\
4.29)\end{array}$ & \\
\hline \multicolumn{4}{|l|}{ Caregiver Characteristics } \\
\hline \multicolumn{2}{|l|}{ Age } & $\begin{array}{l}0.998(0.98- \\
1.02)\end{array}$ & $0.97(0.94-1.00)$ \\
\hline \multirow[t]{2}{*}{ Gender } & Female & 1 & 1 \\
\hline & Male & $\begin{array}{l}0.722(0.39- \\
1.34)\end{array}$ & $0.55(0.24-1.22)$ \\
\hline \multirow[t]{2}{*}{ Employment } & No & 1 & \multirow[t]{2}{*}{$\mathrm{NI}$} \\
\hline & Yes & $\begin{array}{l}0.61(0.33- \\
1.12)\end{array}$ & \\
\hline \multirow[t]{2}{*}{ Caregiver living with patient } & \multirow{2}{*}{$\begin{array}{l}\text { No } \\
\text { Yes }\end{array}$} & $\begin{array}{l}0.711(0.40- \\
1.27)\end{array}$ & \multirow[t]{2}{*}{$\mathrm{NI}$} \\
\hline & & 1 & \\
\hline \multirow[t]{2}{*}{ Level of education } & $\begin{array}{l}\text { Low (Unfinished - } \\
\text { secondary) }\end{array}$ & $\begin{array}{l}0.96(0.54- \\
1.73)\end{array}$ & \multirow[t]{2}{*}{$\mathrm{NI}$} \\
\hline & $\begin{array}{l}\text { High (vocational - } \\
\text { tertiary) }\end{array}$ & 1 & \\
\hline \multirow[t]{3}{*}{ Relationship with patient } & Partner & 1 & \multirow[t]{3}{*}{$\mathrm{NI}$} \\
\hline & Children & $\begin{array}{l}0.93(0.52- \\
1.67)\end{array}$ & \\
\hline & Other & $\begin{array}{l}0.36(0.07- \\
1.80)\end{array}$ & \\
\hline
\end{tabular}




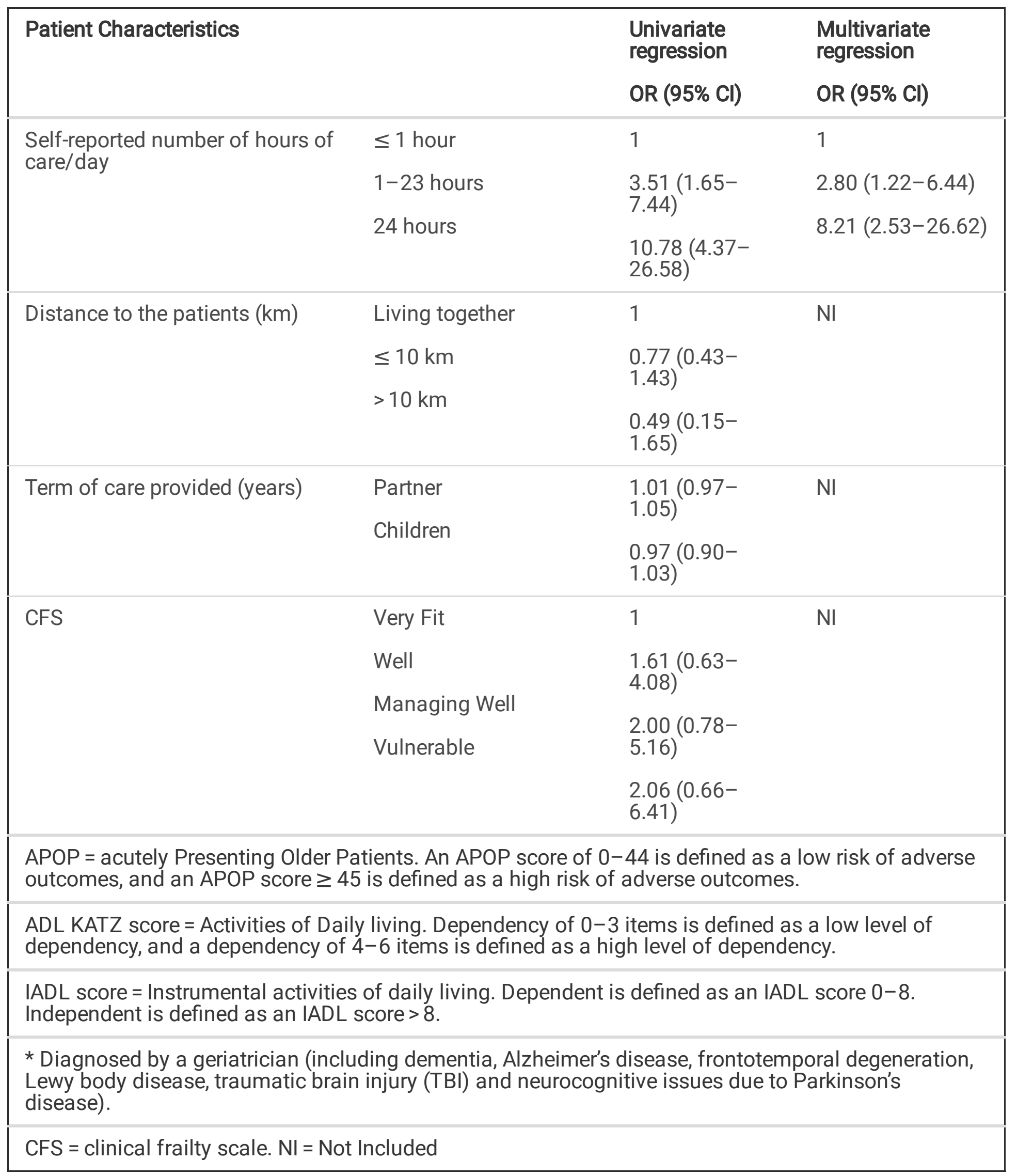

In the multivariate logistic regression analysis, cognitive impairment of the patient (OR = 3.91), IADL dependency $(O R=4.46)$, and self- reported number of hours of care per day by the caregiver $(O R=2.80$ for 
$1-23$ hours vs $<1$ hour and $\mathrm{OR}=8.21$ for 24 hours vs $<1$ hour) were independently associated with high caregiver burden (Table 3 ).

Four weeks after ED presentation, the 78 caregivers with a high burden were reassessed for possible persistence of burden. Of these, six (7\%) were lost to follow-up because they could not be reached by telephone. In 49/78 caregivers (63\%), the high burden persisted.

\section{Discussion}

This is the first study to assess the prevalence and risk factors for caregiver burden of acutely presenting older patients in the ED. Approximately $40 \%$ of the caregivers experienced a high burden. These findings demonstrate that burden is highly prevalent among caregivers presenting at the ED. We identified an association between caregiver burden and patients with cognitive impairment or IADL dependency, as well as the number of self-reported hours of care.

Most prior studies on caregiver burden were conducted on specific patient populations such as older patients with dementia, cancer, or advanced illness. ${ }^{(4,21-23)}$ In our cohort, caregiver burden was relatively high compared to other studies. $(4,5,24)$ This could be explained by several patient and caregiver characteristics. First, our study included a relatively high number of dependent care recipients such as patients with cognitive impairment, stroke survivors and an active malignancy. However, this profile is probably representative for the community dwelling older patients in our society. Second, more than half of the caregivers were female. It has been shown that women more often care for ill relatives and are more likely than men to suffer from depression regarding caregiving tasks. ${ }^{(25)}$ Third, the number of multigenerational caregivers, those who care for children and parents, is rising as life expectancies increase. Increasing workloads and caring for a relative generally negatively affect the daily lives of caregivers and may contribute to their burden. ${ }^{(26)}$ Fourth, the ED visit may have been the climax of a long, debilitating journey of illness and disability, which may be reflected in a high caregiver burden. Finally, the identification of caregivers depends on the definition: caregivers with no burden and few care tasks may not consider themselves as caregiver, which may be associated with an overestimation of the percentage of burden. ${ }^{(27)}$

This study confirms previous research that found a univariate association between caregiver burden and cognitive impairment, ADL dependency, and IADL dependency of patients. ${ }^{(5,25,28,29)}$ After adjusting for the other variables, we found that only cognitive impairment and IADL dependency were associated with a high caregiver burden. This could be explained by the ADL support provided by homecare professionals. Homecare assistance has been reported by $50 \%$ of the participants. IADL includes tasks that are not always resolved by homecare professionals, such as grocery shopping, cooking, and assisting with patient transfers.

Caregiver burden does not only harm the caregiver, but is also associated with potential patient health risks. It may result in inadequate care, medication mistakes, and elder abuse or neglect. ${ }^{(9)}$ Although it is 
likely that caregiver burden is associated with higher ED utilization, this has not been investigated. However, studies on unplanned hospital readmission of older patients found an association between caregiver burden and the risk of readmission. ${ }^{(19)}$ Furthermore, social problems and insufficient social support have been identified as major drivers of ED use. ${ }^{(30)}$

Surprisingly, the patients' children reported more years of caregiving than the patients' partners. They may have considered themselves caregiver sooner than patients' partners. Nevertheless, both the term of care provided and the relationship between the caregiver and patient were not associated with burden.

Approximately $60 \%$ of the caregivers experienced no burden. In fact, caregiving was also associated with positive aspects or a positive appraisal. The caregiver may experience an improved relationship with the patient, a higher self-efficacy, and a sense of personal growth. This may contribute to a better quality of life for the caregiver. ${ }^{(31-33)}$ However, caregivers are often untrained for this challenging role. They may not have access to caregiving information or support, and caregiving education programs are not widely implemented. ${ }^{(34)}$

This study has several limitations. First, the CSI was measured during presentation at the ED, which could have influenced the score. However, the burden persisted in $63 \%$ of the participants four weeks after ED presentation, so this influence was likely limited. Second, some caregivers may have been missed because not all caregivers may consider themselves as such. Caregivers who provide long-term or intensive care more often consider themselves caregiver, which may contribute to overestimation of burden. Various caregiver definitions circulate in the medical literature, further complicating the interpretation of our findings. ${ }^{(35)}$ Third, caregivers were not interviewed about their quality of life or other caregiving experiences. Caregivers may also experience positive aspects of care or positive appraisal such as caregiver satisfaction, but these aspects were not investigated. Fourth, this was a single-center study, and demographics as well as caregiver burden may differ between regions or healthcare systems. Fifth, it was not assessed whether caregivers also had to care for other individuals, which may have contributed to their burden. The persistence of burden was only assessed in caregivers with a CSI $\geq 7$. It is therefore unknown what the course of burden was in the caregivers with a low burden. Finally, 267 of 1,086 patients were not screened for eligibility. In most of the missed cases, the site researcher (T.Z.) was not present and the treating physician did not screen the study patients. Also, ED crowding occurred multiple times during the study period, occasionally hampering inclusion. To reduce selection bias, the researcher was scheduled in random shifts. This "true random sampling" method has been shown to represent the overall population in more than $95 \%$ of the samples and it has a low probability of selection bias. ${ }^{(36)}$

This study's strengths include its prospective design, participant recruitment, extensive sample size of 200 patients, use of a validated caregiver burden scale, inclusion of all older patients referred to the ED regardless of the reason for their ED visit, and the fact that caregivers and patients were interviewed separately. 
Future studies should be multicenter and multinational to assess whether the high caregiver burden occurs in other regions or countries with different healthcare systems. Whether there is an association between high caregiver burden and (low-urgent) ED utilization or negative outcomes should also be ascertained. If an association is found, it should be assessed if the caregiver burden could be used as a screening tool to identify ED patients who are frail or have a high risk of experiencing negative outcomes. Predicting outcomes of older ED patients is essential for delivering adequate care, but can be challenging. (37) New predictors outside the scope of classical medical or frailty-based data may therefore be of extra value. Furthermore, 30-day mortality after ED visit is more associated with frailty than triage urgency. ${ }^{(38)}$ As such, caregiver burden may be a marker of system frailty (as opposed to individual frailty).

\section{Conclusion}

Almost $40 \%$ of older patients in the ED have a caregiver who experiences a high burden. Formal assessment in the ED may help provide adequate care to the patients and their caregivers, but more research is needed to establish whether caregiver burden is associated with frailty or negative outcomes in older ED patients.

\section{Abbreviations}

ED

Emergency department

GPs

General practitioners

ACCI

Age adjusted Charlson Comorbidity index

APOP

Acutely Presenting Older Patients

ADL

Activities of Daily Living

IADL

Instrumental Activities of Daily Living

CFS

Clinical Frailty Scale

CSI

Caregiver strain index

IQR

Interquartile range

ORs

Odds ratios

$\mathrm{Cl}$ 


\section{Declarations}

Ethics approval and consent to participate: This study was approved by the Institutional Review Board of VieCuri Medical Center, Venlo, the Netherlands (\#458).

Consent for publication: not applicable.

Availability of data and materials: please contact corresponding author for data requests.

Conflicts of Interest: The authors have no conflicts of interest to disclose relevant to this article.

Financial disclosure: This study was financed by VieCuri Medical Center's research fund.

Sponsor's Role: the sponsor had no role in the study design, research methods, data collections, analysis, or manuscript preparation.

Author Contributions: T.Z. and D.G.B. designed the study. C.M.H., P.K., L.K., Y.H., R.A.J.K., A.W.L., S.P.M., and M.J. advised on the study design. D.G.B. obtained funding. T.Z. collected data. T.Z. conducted the statistical analyses. M.J. advised on the statistical analyses. T.Z. drafted the article. D.B. advised during the drafting process. C.M.H., P.K., L.K., Y.H., R.A.J.K., A.W.L., S.P.M., and M.J. revised the manuscript for important intellectual content. All of authors approved the current version of the article.

ACKNOWLEDGEMENTS: For editing the English language of the manuscript the authors would like to thank Elsevier Language Editing Services.

\section{References}

1. Verver $D$, Merten $H$, Robben $P$, Wagner $C$. Perspectives on the risks for older adults living independently. Br J Community Nurs. 2017;22(7):338-45.

2. Freedman VA, Spillman BC. Disability and care needs among older Americans. Milbank Q. 2014;92(3):509-41.

3. Verhaegh MTH, Snijders F, Janssen L, Peters N, Mol Y, Kamerman-Celie F, et al. Perspectives on the preventability of emergency department visits by older patients. Neth J Med. 2019;77(9):330-7.

4. Jansen L, Dauphin S, De Burghgraeve T, Schoenmakers B, Buntinx F, van den Akker M. Caregiver burden: An increasing problem related to an aging cancer population. Journal of health psychology. 2019:1359105319893019.

5. Francke ALA. Overbelasting mantelzorger bij dementie neemt toe (2016). NIVEL kennis voor betere zorg. Available at: https://www.nivel.nl/nl/nieuws/overbelasting-mantelzorger-bij-dementie-neemttoe. Accessed december 19, 2019. 
6. Blake H, Lincoln NB, Clarke DD. Caregiver strain in spouses of stroke patients. Clinical rehabilitation. 2003;17(3):312-7.

7. Oliva-Moreno J, Pena-Longobardo LM, Mar J, Masjuan J, Soulard S, Gonzalez-Rojas N, et al. Determinants of Informal Care, Burden, and Risk of Burnout in Caregivers of Stroke Survivors: The CONOCES Study. Stroke. 2018;49(1):140-6.

8. Yu H, Wang X, He R, Liang R, Zhou L. Measuring the Caregiver Burden of Caring for CommunityResiding People with Alzheimer's Disease. PloS one. 2015;10(7):e0132168.

9. Beach SR, Schulz R, Williamson GM, Miller LS, Weiner MF, Lance CE. Risk factors for potentially harmful informal caregiver behavior. J Am Geriatr Soc. 2005;53(2):255-61.

10. Thijssen W, Kraaijvanger N, Barten DG, Boerma MLM, Giesen P, Wensing M. Impact of a welldeveloped primary care system on the length of stay in emergency departments in the Netherlands: a multicenter study. BMC Health Serv Res. 2016;16:149.

11. Kent EE, Rowland JH, Northouse L, Litzelman K, Chou WY, Shelburne N, et al. Caring for caregivers and patients: Research and clinical priorities for informal cancer caregiving. Cancer. 2016;122(13):1987-95.

12. Zachariasse JM, Seiger N, Rood PP, Alves CF, Freitas P, Smit FJ, et al. Validity of the Manchester Triage System in emergency care: A prospective observational study. PloS one. 2017;12(2):e0170811.

13. Charlson ME, Pompei P, Ales KL, MacKenzie CR. A new method of classifying prognostic comorbidity in longitudinal studies: development and validation. Journal of chronic diseases. 1987;40(5):37383.

14. de Gelder J, Lucke JA, Blomaard LC, Booijen AM, Fogteloo AJ, Anten S, et al. Optimization of the APOP screener to predict functional decline or mortality in older emergency department patients: Cross-validation in four prospective cohorts. Experimental gerontology. 2018;110:253-9.

15. Katz S, Ford AB, Moskowitz RW, Jackson BA, Jaffe MW. STUDIES OF ILLNESS IN THE AGED. THE INDEX OF ADL: A STANDARDIZED MEASURE OF BIOLOGICAL AND PSYCHOSOCIAL FUNCTION. Jama. 1963;185:914-9.

16. Lawton MP, Brody EM. Assessment of older people: self-maintaining and instrumental activities of daily living. Gerontologist. 1969;9(3):179-86.

17. Boockvar KS, Meier DE. Palliative care for frail older adults: "there are things I can't do anymore that I wish I could. " Jama. 2006;296(18):2245-53.

18. Robinson BC. Validation of a Caregiver Strain Index. Journal of gerontology. 1983;38(3):344-8.

19. Bonin-Guillaume S, Durand AC, Yahi F, Curiel-Berruyer M, Lacroix O, Cretel E, et al. Predictive factors for early unplanned rehospitalization of older adults after an ED visit: role of the caregiver burden. Aging clinical experimental research. 2015;27(6):883-91.

20. Sullivan MT. Caregiver Strain Index (CSI). Home Healthc Nurse. 2003;21(3):197-8. 
21. Garlo K, O'Leary JR, Van Ness PH, Fried TR. Burden in caregivers of older adults with advanced illness. J Am Geriatr Soc. 2010;58(12):2315-22.

22. Covinsky KE, Newcomer R, Fox P, Wood J, Sands L, Dane K, et al. Patient and caregiver characteristics associated with depression in caregivers of patients with dementia. J Gen Intern Med. 2003;18(12):1006-14.

23. Lilly MB, Robinson CA, Holtzman S, Bottorff JL. Can we move beyond burden and burnout to support the health and wellness of family caregivers to persons with dementia? Evidence from British Columbia. Canada Health social care in the community. 2012;20(1):103-12.

24. Feiten en cijfers over mantelzorgs. 2019. In voor mantelzorg (online). Available at: https://www.invoormantelzorg.nl/nieuws/feiten-en-cijfers-over-mantelzorgers/. Accessed February 4, 2020.

25. Schoenmakers B, Buntinx F, Delepeleire J. Factors determining the impact of care-giving on caregivers of elderly patients with dementia. A systematic literature review. Maturitas. 2010;66(2):191-200.

26. Dukhovnov D, Zagheni E. WHO TAKES CARE OF, WHOM IN THE U.S.? EVIDENCE FROM MATRICES OF TIME TRANSFERS BY AGE AND SEX. Population development review. 2015;41(2):183-206.

27. Institute of Medicine. Patients and informal caregivers. In: Retooling for an Aging America. In: Building the Health Care Workforce. Washington, DC: National Academies Press; 2008.

28. Dauphinot V, Ravier A, Novais T, Delphin-Combe F, Mouchoux C, Krolak-Salmon P. Risk Factors of Caregiver Burden Evolution, for Patients With Subjective Cognitive Decline or Neurocognitive Disorders: A Longitudinal Analysis. J Am Med Dir Assoc. 2016;17(11):1037-43.

29. Kang HS, Myung W, Na DL, Kim SY, Lee JH, Han SH, et al. Factors associated with caregiver burden in patients with Alzheimer's disease. Psychiatry investigation. 2014;11(2):152-9.

30. Burgdorf J, Mulcahy J, Amjad H, Kasper JD, Covinsky K, Wolff JL. Family Caregiver Factors Associated With Emergency Department Utilization Among Community-Living Older Adults With Disabilities. Journal of primary care community health. 2019;10:2150132719875636.

31. Kajiwara K, Nakatani H, Ono M, Miyakoshi Y. Positive appraisal of in-home family caregivers of dementia patients as an influence on the continuation of caregiving. Psychogeriatrics: the official journal of the Japanese Psychogeriatric Society. 2015;15(1):26-31.

32. Semiatin AM, O'Connor MK. The relationship between self-efficacy and positive aspects of caregiving in Alzheimer's disease caregivers. Aging Ment Health. 2012;16(6):683-8.

33. Kate N, Grover S, Kulhara P, Nehra R. Positive aspects of caregiving and its correlates in caregivers of schizophrenia: a study from north India. East Asian archives of psychiatry: official journal of the Hong Kong College of Psychiatrists = Dong Ya jing shen ke xue zhi : Xianggang jing shen ke yi xue yuan qi kan. 2013;23(2):45-55.

34. Peterson K, Hahn H, Lee AJ, Madison CA, Atri A. In the Information Age, do dementia caregivers get the information they need? Semi-structured interviews to determine informal caregivers' education needs, barriers, and preferences. BMC Geriatr. 2016;16(1):164. 
35. Roth DL, Fredman L, Haley WE. Informal caregiving and its impact on health: a reappraisal from population-based studies. Gerontologist. 2015;55(2):309-19.

36. Valley MA, Heard KJ, Ginde AA, Lezotte DC, Lowenstein SR. Observational studies of patients in the emergency department: a comparison of 4 sampling methods. Ann Emerg Med. 2012;60(2):139 45.e1.

37. Carpenter CR, Mooijaart SP. Geriatric Screeners 2.0: Time for a Paradigm Shift in Emergency Department Vulnerability Research. Journal of the American Geriatrics Society. 2020.

38. Blomaard LC, Speksnijder C, Lucke JA, de Gelder J, Anten S, Schuit SCE, et al. Geriatric Screening, Triage Urgency, and 30-Day Mortality in Older Emergency Department Patients. Journal of the American Geriatrics Society. 2020.

\section{Figures}

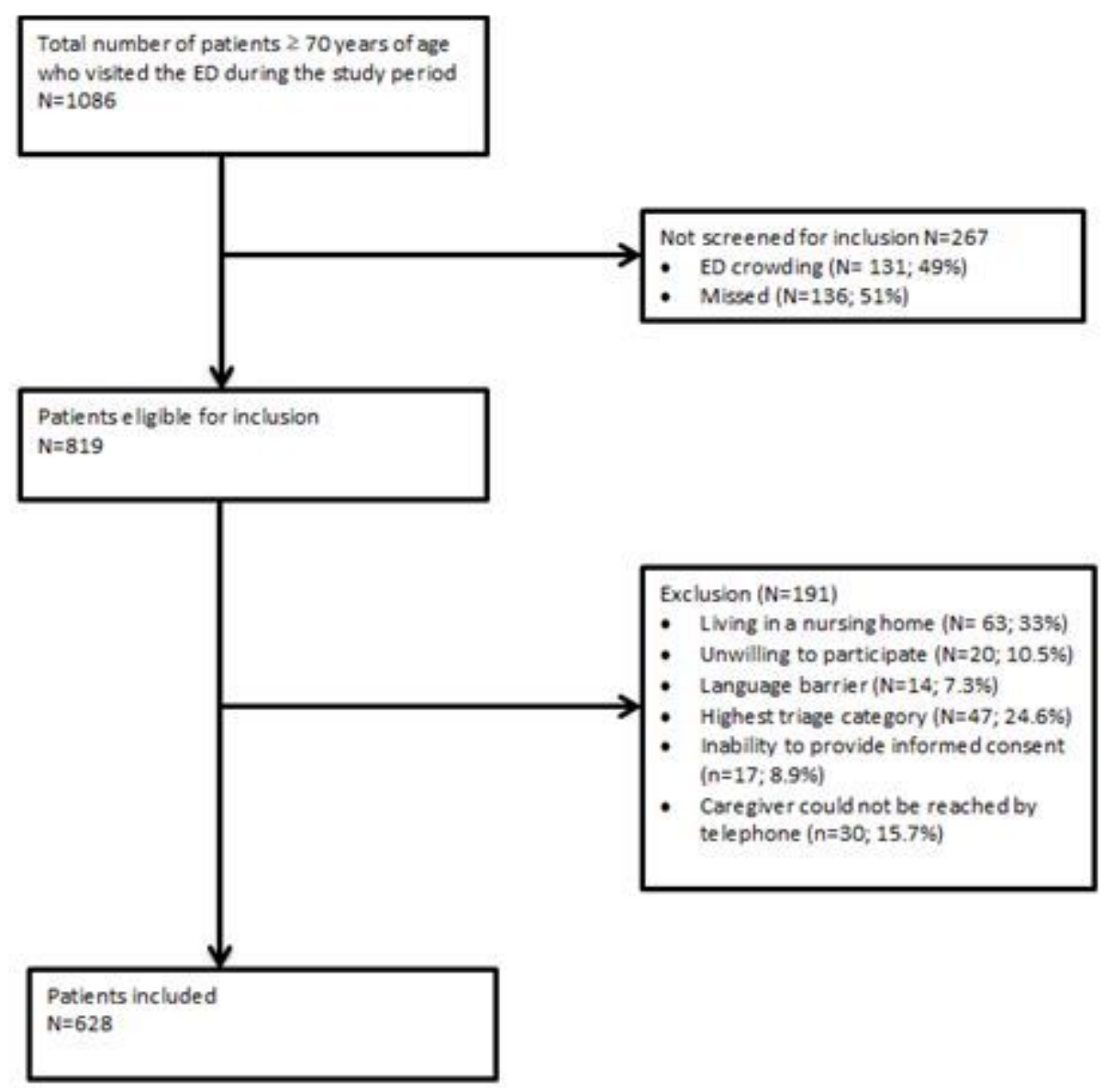

\section{Figure 1}

Patient inclusion and exclusion flow chart. 